\title{
Developing a Training Module in Rigor and Reproducibility in Imaging Sciences
}

\author{
A.M. Medina-Lopez ${ }^{1,2}$, H. Shinogle-Decker ${ }^{1,2}$, N. Martinez-Rivera ${ }^{1,2}$ and E. Rosa-Molinar ${ }^{1,2}$ \\ ${ }^{1 .}$ Microscopy and Analytical Imaging Resource Core Laboratory, University of Kansas, Lawrence, KS \\ 2. Department of Pharmacology and Toxicology and Neuroscience Graduate Program, University of \\ Kansas, Lawrence, KS
}

Researchers' recurrent struggles to compare and reproduce published research results prompted the primary federal funding agencies (e.g., NIH, NSF), scientific journals, press and ethic integrity forums, among others, to discuss scientific experiments' rigor and reproducibility as key factors in credibility of research, and, therefore, as essential elements in teaching and training future scientists. NIH was one of the first agencies to develop a set of guidelines to assure scientific integrity through rigor and reproducibility ${ }^{[1]}$. The guidelines focus on the rigor required in four areas: reviewing the strengths and weakness of prior research and in developing the scientific premise; developing a research design based on the scientific method to achieve "robust and unbiased" results; delineating and explaining relevant variables such as, for example, sex, age, etc. that could make a difference in the outcomes; and, authenticating and validating chemical and biological reagents. ${ }^{[1]}$

In view of the NIH guidelines, we determined to promote scientific integrity, accountability, and responsibility in imaging science by teaching young scientists (i.e., high schoolers, undergrads, and others) the importance of rigor and reproducibility in imaging science. Thus, we began to develop training modules in immunohistochemistry and microscopy techniques.

Our ultimate goal was to develop a module for imaging sciences (Fig. 1). To lay the foundation for the module, we applied the four NIH guidelines to published articles and to our attempts to replicate results. We analyzed the articles according to NIH guidelines for rigor and reproducibility and then attempted to replicate the reported results. In our work, we first identified our scientific premise: the authentication and validation of cell lines and key reagents for studying Golgi complex in mammalian fibroblasts. Subsequently, we constructed our experimental design in keeping with the article(s) whose results we attempted to replicate and compared the results. In addition, we took into account the relevant variables to validate our reagents.

In our attempts to authenticate and validate cell lines and key reagents used in the published studies, we encountered three problems: 1) the effects of Brefeldin A on Golgi complex; 2) Brefeldin A effects on cell behavior; and 3) Golgi complex staining with C6 NBD Ceramide. Our work in attempting to replicate results/data demonstrates that the articles' lack of details about choice, source, and preparation of reagents; source and characterization of antibodies; positive and negative controls; and settings, calibrations, and standards of instruments, made it impossible to duplicate the reported results. Results revealed that temperature and permeabilization variables, but not fixation, affect the Golgi stain signal from the samples by either enhancing or reducing it; these experimental details were not reported in the original article(s). Moreover, our results for the effects of BFA were contrary to reported results. After testing all different concentrations of BFA, we found no effect of it on cell structure and motility; no difference in Golgi labeling, and no inhibition in movement and pseudopod formation. This work demonstrates how published data that lack details and essential information regarding methodology could delay or counteract the progress of other ongoing research. 
The foundational work for the development of a module for imaging sciences shows how lack of transparency in materials and methods could impact related research and points to the need for precision, thoroughness, and rigor in delineating details essential to data reproducibility [2-3] [4].

\section{References:}

[1] NIHReproducibilityEfforts. (2016, 9 30). Rigor and Reproducibility. Retrieved from NIH Grants and Funding: https://grants.nih.gov/reproducibility/index.htm

[2] Marcus, E. Credibility and Reproducibility. Cell (2014), p. 965.

[3] Vitek, J., \& Kilibera, T. EMSOFT '11 Proceedings of the ninth ACM international conference on Embedded software (2011) p. 33. Taipei, Taiwan : ACM.

[4] Research partially supported by grants to ER-M from NIH (GM-115042; GM-078441; MH-106245) and NSF (HRD-1137725).

Problem 1: Brefeldin A effects in the Golgi complex

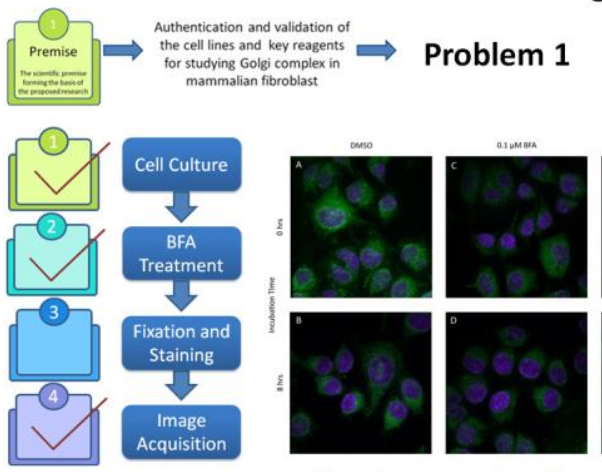

Result 1: Disruption of Golgi complex due to Brefeldin A effects

Problem 3: Golgi complex staining with C6 NBD Ceramide
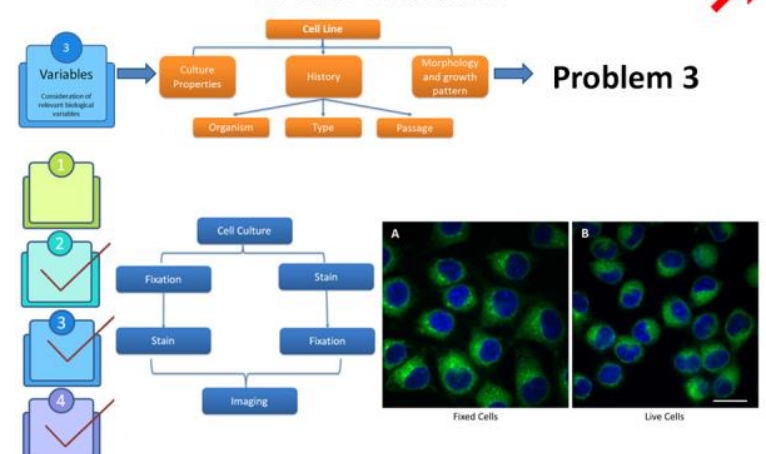

Result 3: $C_{6}$ NBD Ceramide for staining Golgi complex
Problem 2: Brefeldin A effects in cell behavior
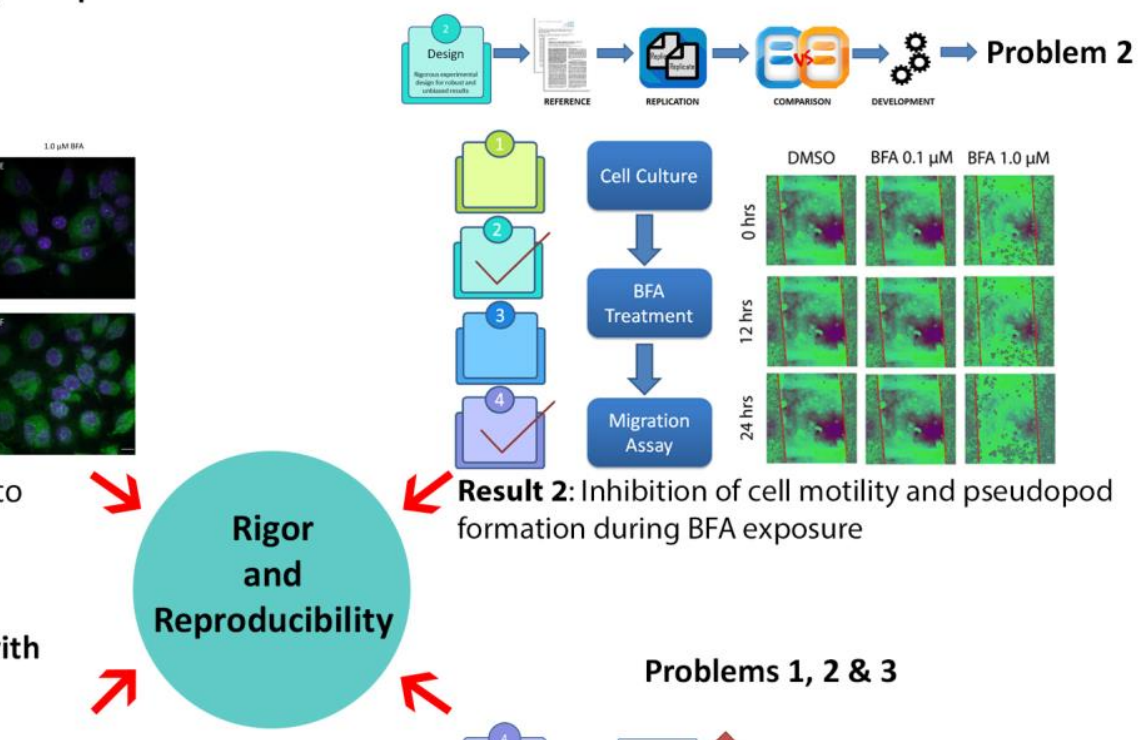

Result 2: Inhibition of cell motility and pseudopod formation during BFA exposure

Problems 1, $2 \& 3$

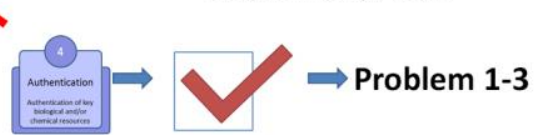

Figure 1. Development of the module for imaging sciences

Scale bar $50 \mu \mathrm{m}$ 